NBER WORKING PAPER SERIES

\title{
SCARCITY OF IDEAS AND R\&D OPTIONS: USE IT, LOSE IT OR BANK IT
}

\author{
Nisvan Erkal \\ Suzanne Scotchmer \\ Working Paper 14940 \\ http://www.nber.org/papers/w14940
NATIONAL BUREAU OF ECONOMIC RESEARCH
1050 Massachusetts Avenue
Cambridge, MA 02138

May 2009

This paper expands and supersedes the first part of our 2007 paper "Scarcity of Ideas and Options to Invest in R\&D." We especially thank Jennifer Reinganum for her comments at the Fifth Summer Workshop in Industrial Organization in Auckland. We also thank Michael Katz, Stephen Maurer, Robert Merges, Deborah Minehart, Paul Seabright, Brian Wright and participants of the 2008 Research Symposium on the Economics and Law of the Entrepreneur organized by the Searle Center on Law, Regulation, and Economic Growth for useful discussion. We thank the Toulouse Network on Information Technology, NSF Grants SES 0531184, 0830186, and Australian Research Council Grant DP0987070 for financial support. Nisvan Erkal thanks the University of California, Berkeley (ARE), for hosting her as a visiting scholar.Email: n.erkal@unimelb.edu.au, scotch@berkeley.edu. The views expressed herein are those of the author(s) and do not necessarily reflect the views of the National Bureau of Economic Research.

NBER working papers are circulated for discussion and comment purposes. They have not been peerreviewed or been subject to the review by the NBER Board of Directors that accompanies official NBER publications.

(C) 2009 by Nisvan Erkal and Suzanne Scotchmer. All rights reserved. Short sections of text, not to exceed two paragraphs, may be quoted without explicit permission provided that full credit, including (c) notice, is given to the source. 
Scarcity of Ideas and R\&D Options: Use it, Lose it or Bank it

Nisvan Erkal and Suzanne Scotchmer

NBER Working Paper No. 14940

May 2009

JEL No. K00,L00,O34

\begin{abstract}
$\underline{\text { ABSTRACT }}$
We investigate rewards to $R \& D$ in a model where substitute ideas for innovation arrive to random recipients at random times. By foregoing investment in a current idea, society as a whole preserves an option to invest in a better idea for the same market niche, but with delay. Because successive ideas may occur to different people, there is a conflict between private and social optimality. We characterize the welfare-maximizing reward structure when the social planner learns over time about the arrival rate of ideas, and when private recipients of ideas can bank their ideas for future use. We argue that private incentives to create socially valuable options can be achieved by giving higher rewards where "ideas are scarce."
\end{abstract}

Nisvan Erkal

Department of Economics

University of Melbourne

VIC 3010 Australia

n.erkal@unimelb.edu.au

Suzanne Scotchmer

Department of Economics

Evans Hall

University of California

Berkeley, CA 94720-3880

and NBER

scotch@berkeley.edu 


\section{Introduction}

Rewards for R\&D should depend on the underlying innovative process. We consider an innovative environment where different agents have substitute ideas for how to fill a given market niche. The purpose of the reward system is to choose among the substitute ideas.

Many models of R\&D begin from a concept that opportunities are common knowledge and eternally present, but progress can nevertheless be slow because R\&D is costly and resources are scarce. ${ }^{1}$ However, there is another reason that progress can be slow: Ideas for investment are themselves scarce, not only from an individual's point of view, but also for society as a whole. Even if a market niche or economic need is known, there may be considerable delay before someone realizes how to fill it at reasonable cost. In addition to the scarcity of resources, the scarcity of ideas is another constraint on progress.

In the innovative environment we study, investment opportunities are not common knowledge. Ideas for how to fill the market niche have random cost, and arrive at random times to random individuals. If the recipient of an idea invests the cost, the idea becomes an innovation that fills the market niche. Most importantly, innovative environments are distinguished by the arrival rate (scarcity) of ideas.

Our objective is to show how rewards should reflect the scarcity of ideas. The social planner does not know the ideas that have arrived, and does not know who received them. If all ideas were available at the same time, the goal of the social planner would be to find the minimum cost idea. However, that is not possible because the ideas arrive at random times. The planner can weed out high-cost ideas by offering limited rewards, but he still faces a trade-off between cost and delay. We characterize the reward structure that minimizes the burden of these costs. In this reward structure,

- rewards increase with the scarcity of ideas;

- rewards increase with delay in filling the market niche; and

\footnotetext{
${ }^{1}$ This is implicitly the premise of a large literature on patent races that builds on models surveyed by Reinganum (1989).
} 
- the profit on $\mathrm{R} \& \mathrm{D}$ investments will be positive in equilibrium due to the scarcity of ideas.

These conclusions are tied to the notion that ideas are scarce, so that the reward policy must mediate between cost and delay. Higher rewards reduce delay by encouraging investment in higher-cost ideas instead of waiting for lower-cost ideas. The first point states that this is welfare-maximizing when ideas arrive at long intervals ("ideas are scarce"). The second point arises when the scarcity (arrival rate) of ideas is unknown. As time passes with no innovation, observers begin to think that the arrival rate of ideas is low. It becomes even more attractive to tolerate higher cost in order to reduce delay. We do not know of other papers where the reward policy changes dynamically. ${ }^{2}$

As in O'Donoghue, Scotchmer and Thisse (OST, 1998) and Scotchmer (1999), our model distinguishes between ideas and innovations. However, delay is never optimal in these papers. OST (1998) address environments where the ideas are complements in the sense that each idea builds on previous ideas, and Scotchmer (1999) addresses environments where ideas serve different market niches, but there are no substitute ideas for a given market niche. In the model we discuss here, it is because ideas are substitutes that a certain amount of delay should be tolerated. One of the ideas that arrives during the delay may have low cost.

Our model is a real options model in the spirit of MacDonald and Siegel (1986) and Dixit and Pindyck (1994). An investment is irreversible and could turn out to be a mistake. To avoid mistakes, there is a value to delay. In many real options models, the value of the option is internalized by the firm. In our model, ideas (investment opportunities) accrue to random firms, which means that although waiting is valuable to society, the value of waiting is not internalized by any potential innovator. The problem of the social planner is to ensure that private recipients of ideas preserve socially valuable options.

\footnotetext{
${ }^{2}$ A dynamically changing reward policy would presumably be optimal in any model where learning takes place about something relevant to rewards. Although the authors do not analyze this aspect, two papers where that might be true are Choi (1991) and Malueg and Tsutsui (1997). In those papers, there is an unknown parameter that governs the hazard rate of success in a production function for $R \& D$ that is common knowledge among the firms in a race. In our model below, the planner is learning about the hazard rate at which the population as a whole receives ideas for investment. There is no commonly known but uncertain production function for R\&D.
} 
Our modeling apparatus is reminiscent of search models (see McCall and McCall, 2008), although we do not interpret our random process as search. In search models, all opportunities arrive to a single searcher who sets an optimal stopping policy. In our model, ideas are so scarce that no individual is likely to receive more than one idea. The planner knows that the population as a whole is receiving ideas, and knows something about the stochastic process, but does not know who receives ideas or when. Nevertheless, the planner must set the reward policy. Despite this fundamental difference, the planner's reward policy in the case of a known arrival rate is similar to the stopping rule that emerges in search models. Although some search models involve learning, we do not know of results in the search literature that are analogous to our results for the case that the planner is learning about the arrival rate.

The paper is structured as follows. In section 2, we set forth a simple model of scarce ideas. In sections 3 and 4 , we characterize the cost thresholds that maximize social welfare. At each date, the cost threshold is a cost such that the possessor of a lower-cost idea should invest. The planner realizes that if someone invests, society is giving up an option, namely, the option to wait for a better idea. The option that is preserved by not investing is a social option, not internalized by any potential investor.

The stochastic process that determines the option value depends on whether rejected ideas are lost forever ("use it or lose it") or banked for future use ("use it or bank it"). We treat these two cases in sections 3 and 4 . Banking is attractive to the social planner when he does not know the arrival rate of ideas. As time passes, the planner becomes more pessimistic about the arrival rate. An idea that seemed too costly a year ago will seem more attractive at present because more delay is predicted. The planner will therefore want access to the banked idea with lowest cost.

In section 5, we characterize how cost thresholds can be implemented, with and without banking of ideas. The reward policy that implements a given cost threshold must account for the strategic choices of idea recipients. Banking is attractive to the recipient of an idea whenever the reward function is increasing. The recipient of the idea may be willing to 
forego the profit available by investing at present in order to gamble on a higher reward in the future. Of course, the recipient may be preempted in the meantime.

We conclude in section 6 by mentioning some ways that the reward policies described in section 5 correspond to legal institutions.

\section{A Model of Scarce Ideas}

We assume there is market niche that may be filled with an innovation. The social value of filling the market niche is $v / r$, where $r$ is the discount rate.

There is an exogenous process by which the potential innovators receive ideas for filling this market niche. To innovate, the inventor must first have an idea, which we interpret as an act of imagination, and then have an incentive to invest in it. Each idea occurs at a random time, to a random recipient. Each idea has associated to it an $R \& D$ cost that is drawn independently from a common distribution $F$ with support in $[0, \infty)$ and density $f$. To create an innovation, the recipient of an idea must invest the cost. We assume that the ideas rain down on the population as a whole according to a Poisson process with parameter

$\lambda$, and we take the parameter $\lambda$ as a measure of scarcity. If the hit rate $\lambda$ is low, ideas are scarce.

The recipient of an idea can invest in it, discard it, or bank it, which means to remember it for future use. If the recipient of an idea invests in it, the process stops because the market niche has been filled. The reward policy will therefore operate by getting the population of potential innovators to screen their ideas and then to discard or bank those with costs that are too high. The value of the social option created by not investing is that another idea might entail a lower cost. There is thus a social trade-off between cost and delay. The policy objective is to manage this trade-off in a way that maximizes social welfare.

We assume that each agent receives at most one idea. This is an intentionally extreme assumption that highlights the main premise of the paper. Ideas are scarce, not only for society as a whole, but especially from the perspective of any individual.

The social policy is described by a threshold function $c: \mathbf{R}_{+} \rightarrow \mathbf{R}_{+}$such that the 
recipient of an idea at time $t$ invests if the cost of the idea is less than $c(t)$. A threshold function is stationary if there exists $\bar{c}$ in $\mathbf{R}_{+}$such that $c(t)=\bar{c}$ for all arrival times $t$. We say an idea at time $t$ is viable if it has cost less than $c(t)$. The expected cost of a random viable idea that arrives at time $t$ is

$$
E_{F}(c(t))=\int_{0}^{c(t)} \hat{c} \frac{f(\hat{c})}{F(c(t))} d \hat{c}
$$

We say that the investment process survives to $t$ if there is no viable idea before $t$.

We now consider two versions of the ideas process. In the "use it or lose it" model, an idea that is not used immediately is lost. For example, an idea may be lost or forgotten if the recipient moves on to other projects. However, not all ideas will be lost, especially if there is an incentive to remember them. We consider this in the "use it or bank it" model. The truth is probably somewhere between these two models for most $R \& D$ environments. We study the two extreme cases in order to show their implications for the reward policy.

\section{Use it or lose it}

In this section, we assume that, if the recipient of an idea decides not to invest, the idea is lost to everyone, including the recipient, and cannot be reclaimed later.

Let $P(t \mid \lambda, c)$ be the probability of surviving to time $t$, as seen from time 0 , when the threshold function is $c$ and the arrival rate of ideas is $\lambda$. The survival probability $P$ differs according to whether ideas can be banked, but in both models, the probability distribution on survival times is stochastically larger at smaller arrival rates.

When recipients either use their ideas or forget them immediately, the instantaneous arrival rate of viable ideas at time $t$ is $\lambda F(c(t))$. As seen from time $t=0$, the probability of survival to time $t$ with no viable idea is $P(t \mid \lambda, c)$, defined as follows.

$$
P(t \mid \lambda, c)=e^{-\Lambda(t, c)} \text { where } \Lambda(t, c)=\int_{0}^{t} \lambda F(c(\tilde{t})) d \tilde{t}
$$

(See, for example, Snyder and Miller, 1991, p. 51.) The probability of surviving to $\hat{t}$, conditional on surviving to an earlier time $t$, is $P(\hat{t} \mid \lambda, c) / P(t \mid \lambda, c)$. As seen from time $t$, 
the probability that the first viable idea arrives at $\hat{t}>t$ is

$$
\frac{d}{d \hat{t}}\left[1-\frac{P(\hat{t} \mid \lambda, c)}{P(t \mid \lambda, c)}\right]=\lambda F(c(\hat{t})) e^{-\Lambda(\hat{t}, c)+\Lambda(t, c)}
$$

We study both the case that the arrival rate $\lambda$ is known, and the case that the arrival rate is unknown. We now define a social welfare function that reflects the tradeoff between cost and delay, and characterize the threshold function that maximizes it. We show for the case when $\lambda$ is known, that the threshold function is stationary, and further, that the optimal stationary value decreases with the arrival rate of ideas, $\lambda$.

Conditional on an arbitrary threshold function $c$, and assuming that no viable idea has occurred before $t$, social welfare measured from time $t$ is $V$, defined by

$$
\begin{aligned}
V(t, c, \lambda) & =\int_{t}^{\infty} e^{-r(\hat{t}-t)}\left(\frac{v}{r}-E_{F}(c(\hat{t}))\right) \lambda F(c(\hat{t})) e^{-\Lambda(\hat{t}, c)+\Lambda(t, c)} d \hat{t} \\
& =\int_{t}^{\infty} e^{-r(\hat{t}-t)}\left(\frac{v}{r}-E_{F}(c(\hat{t}))\right) \lambda F(c(\hat{t})) \frac{P(\hat{t} \mid \lambda, c)}{P(t \mid \lambda, c)} d \hat{t}
\end{aligned}
$$

If the threshold function $c$ maximizes welfare, the following condition holds at each $t$.

$$
\left(\frac{v}{r}-c(t)\right)=V(t, c, \lambda)
$$

The left hand side is the net social value of investing in the threshold idea at time $t$. The right hand side is the expected, discounted value of waiting for a better idea. If the left hand side were greater than the right hand side, then social welfare could be improved by increasing the threshold cost. If the right hand side were greater than the left hand side, then social welfare could be increased by decreasing the threshold cost.

It is well known in the search literature that, because this is a stationary problem, the optimal threshold is a stationary value, say $\bar{c}$. Welfare as a function of the stationary threshold $\bar{c}$ can be written as

$$
\begin{aligned}
\bar{V}(t, \bar{c}, \lambda) & =\left(\frac{v}{r}-E_{F}(\bar{c})\right) \int_{t}^{\infty} e^{-r(\hat{t}-t)} \lambda F(\bar{c}) e^{-\lambda F(\bar{c})(\hat{t}-t)} d \hat{t} \\
& =\left(\frac{v}{r}-E_{F}(\bar{c})\right) \frac{\lambda F(\bar{c})}{\lambda F(\bar{c})+r}
\end{aligned}
$$


This expression shows the trade-off faced by the policy maker. If a higher stationary cost threshold $\bar{c}$ is tolerated, the innovation will arrive sooner since the hit rate of viable ideas, $\lambda F(\bar{c})$, is then higher, and the discounting expression, $\frac{\lambda F(\bar{c})}{(\lambda F(\bar{c})+r)}$, is larger.

Since the optimal threshold function is stationary, we can conceive of it as a value $c^{*}(\lambda) \in \mathbf{R}_{+}$, where $c(t)=c^{*}(\lambda)$ for each $t$. The first-order condition for maximizing (6) can be written for each $\lambda$ as

$$
\left(\frac{v}{r}-c^{*}(\lambda)\right)-\frac{\lambda F\left(c^{*}(\lambda)\right)}{\left(\lambda F\left(c^{*}(\lambda)\right)+r\right)}\left(\frac{v}{r}-E_{F}\left(c^{*}(\lambda)\right)\right)=0 .
$$

The (unique) solution $c^{*}(\lambda)$ has the property that investing in the marginal innovation today, and receiving net value $\left(\frac{v}{r}-c^{*}(\lambda)\right)$, is as valuable as waiting for the next viable idea. The next viable idea will arrive with delay, but may have a lower cost. With a higher arrival rate $\lambda$, it makes sense to wait for a lower resource cost, because the time between ideas is short.

We summarize these conclusions in the following proposition. Part (a) is proved in the appendix in a different way than in the search literature. Part (b) follows from differentiating (7) implicitly.

Proposition 1 Suppose that the recipient of an idea must use it or lose it. Suppose that the arrival rate of ideas, $\lambda$, is fixed and known. Then (a) given $\lambda$, the welfare-maximizing cost threshold is stationary; and (b) the welfare-maximizing stationary threshold $c^{*}(\lambda)$ is decreasing with $\lambda$.

We now turn to the more realistic case that $\lambda$ is unknown. Like all contracts, R\&D incentives must depend on things that are verifiable. A prize or patent authority knows whether the market niche has been filled, but does not observe the hypothetical distribution of arrival times, and does not observe the arrival of ideas that are rejected.

The length of time without arrival of a viable idea is a signal of $\lambda$. A long period with no arrival should make the observer more pessimistic about $\lambda$ - it shifts the posterior distribution on $\lambda$ toward lower values. However, the posterior distribution on $\lambda$ must also 
account for the fact that some ideas are rejected. Thus, the threshold function for accepting or rejecting ideas is an ingredient to forming a posterior belief on $\lambda$.

We show that, when the posterior distribution on $\lambda$ is changing as time passes, neither the optimized value function nor the optimizing threshold function is stationary. Because the posterior distribution on $\lambda$ shifts toward lower values as time passes with no viable idea, the (optimized) value of waiting for a better idea decreases with time. This implies that society should be less discriminating about which idea is accepted. In particular, the preferred cost threshold is increasing instead of being stationary.

Let $\tilde{h}$ be the prior density function for the distribution of $\lambda$ with support $[0, \infty)$. Then the posterior density, conditional on a threshold function $c$, and conditional on no viable hit having arrived by time $\hat{t}$, is $h(\cdot \mid \hat{t}, c)$ with cumulative distribution $H(\cdot \mid \hat{t}, c)$, where $h(\cdot \mid \hat{t}, c)$ satisfies

$$
h(\lambda \mid \hat{t}, c)=\frac{\tilde{h}(\lambda) P(\hat{t} \mid \lambda, c)}{\int \tilde{h}(\lambda) P(\hat{t} \mid \lambda, c) d \lambda} \text { for each } \lambda \in(0, \infty)
$$

The posterior depends on the threshold function $c$ up to time $\hat{t}$, through the values of $P(\cdot \mid \lambda, c)$, and more specifically in the "use it or lose it" model, through $\Lambda(\hat{t}, c)$. Let $E(\lambda \mid \hat{t}, c)$ be the expected value of $\lambda$ :

$$
E(\lambda \mid \hat{t}, c)=\int_{0}^{\infty} \lambda h(\lambda \mid \hat{t}, c) d \lambda
$$

Lemma 1 If $t_{1}<t_{2}$, the distribution $H\left(\cdot \mid t_{1}, c\right)$ stochastically dominates $H\left(\cdot \mid t_{2}, c\right)$. Moreover, $E(\lambda \mid t, c)$ decreases with $t$.

Seen from $t=0$, and accounting for the uncertainty on $\lambda$, the probability of survival to time $t$ is

$$
\tilde{P}(\hat{t} \mid c)=\int_{0}^{\infty} P(\hat{t} \mid \lambda, c) \tilde{h}(\lambda) d \lambda=\int_{0}^{\infty} e^{-\Lambda(\hat{t}, c)} \tilde{h}(\lambda) d \lambda
$$

Similarly, the probability of surviving to $\hat{t}$, conditional on surviving to $t$ is

$$
\frac{\tilde{P}(\hat{t} \mid c)}{\tilde{P}(t \mid c)}=\int_{0}^{\infty} \frac{P(\hat{t} \mid \lambda, c)}{P(t \mid \lambda, c)} h(\lambda \mid t, c) d \lambda=\int_{0}^{\infty} e^{-\Lambda(\hat{t}, c)+\Lambda(t, c)} h(\lambda \mid t, c) d \lambda
$$


The probability that the first viable idea arrives at $\hat{t}$, conditional on surviving to $t<\hat{t}$, is given by

$$
\frac{d}{d \hat{t}}\left[1-\frac{\tilde{P}(\hat{t} \mid c)}{\tilde{P}(t \mid c)}\right]=\frac{F(c(\hat{t})) \int \lambda e^{-\Lambda(\hat{t}, c)} \tilde{h}(\lambda) d \lambda}{\tilde{P}(t \mid c)}=\frac{\tilde{P}(\hat{t} \mid c)}{\tilde{P}(t \mid c)} F(c(\hat{t})) E(\lambda \mid \hat{t}, c)
$$

The social value of continuing from time $t$ is given by a function $\tilde{V}$ defined in the first line of (10). Substituting for $V(t, c, \lambda)$ from (4) gives the expression in the second line, which shows more explicitly the probabilities of investing at each time $\hat{t}$, as they depend on the underlying $\lambda$. The rewriting in the third line focuses on the fact that it is the beliefs $E(\lambda \mid \hat{t}, c)$ that matter at each $\hat{t}$. The instantaneous probability of receiving a viable idea is $E(\lambda \mid \hat{t}, c) F(c(\hat{t}))$. As seen from $t$, the probability of arriving at time $\hat{t}$ in the first place is $\frac{\tilde{P}(\hat{t} \mid c)}{\tilde{P}(t \mid c)}$.

$$
\begin{aligned}
\tilde{V}(t, c, \tilde{h}) & =\int_{0}^{\infty} V(t, c, \lambda) h(\lambda \mid t, c) d \lambda \\
& =\int_{t}^{\infty} e^{-r(\hat{t}-t)}\left(\frac{v}{r}-E_{F}(c(\hat{t}))\right) \int_{0}^{\infty} \lambda F(c(\hat{t})) e^{-[\Lambda(\hat{t}, c)-\Lambda(t, c)]} h(\lambda \mid t, c) d \lambda d \hat{t} \\
& =\int_{t}^{\infty} e^{-r(\hat{t}-t)}\left(\frac{v}{r}-E_{F}(c(\hat{t}))\right) E(\lambda \mid \hat{t}, c) F(c(\hat{t})) \frac{\tilde{P}(\hat{t} \mid c)}{\tilde{P}(t \mid c)} d \hat{t}
\end{aligned}
$$

Let $c: \mathbf{R}_{+} \rightarrow \mathbf{R}_{+}$be the threshold function that maximizes $\tilde{V}(0, \cdot \tilde{h})$. Then, analogous to (5), the welfare-maximizing threshold function $c$ satisfies the following at each $t$ :

$$
\frac{v}{r}-c(t)=\tilde{V}(t, c, \tilde{h})
$$

To show that the optimizing $c$ is increasing, it is enough to show that $\tilde{V}(\cdot, c, \tilde{h})$ is decreasing. Intuitively, $\tilde{V}$ is decreasing because the observer becomes more and more pessimistic about the arrival rate of ideas as time continues without a viable hit. Because of this pessimism, more delay is expected. Delay can be mitigated by tolerating higher cost.

In the appendix we prove the following result.

Proposition 2 Suppose that the recipient of an idea must use it or lose it. Suppose that the arrival rate of ideas, $\lambda$, has a prior distribution $\tilde{h}$ with support $[0, \infty)$. Let $c$ be the threshold function that maximizes $\tilde{V}(0, \cdot, \tilde{h})$. Then $c$ is increasing. 
In section 5, we discuss how the welfare-maximizing cost threshold can be implemented. In the "use it or lose it" model, the threshold is implemented by setting the reward equal to the threshold. This is because each recipient of an idea has a single opportunity to invest. He will not receive another idea (ideas are scarce), and he must either invest in the idea immediately or lose it forever.

\section{Use it or Bank it}

When the reward is equal to the cost threshold, and therefore (with unknown $\lambda$ ) increasing, the possessor of an idea may have an incentive to delay investment to get a higher reward. If the recipient can bank his idea for later use, the social planner needs to take this into account in choosing his preferred cost threshold, as well as a reward policy to implement it.

How should the planner view banking? The social planner does not want to delay investments that should be viable under his preferred cost threshold. His reward function should ensure that this does not happen. At the same time, banking ideas for future use is tantamount to increasing the arrival rate of ideas in the future. Since this is valuable, the cost threshold should take it into account.

With banking, the social policy is again a threshold function $c$. Ideas accumulate over time and are banked by the recipients. An idea that is converted to an innovation can either be a banked idea or a new idea.

The marginal probability of investment at time $t$ must be described differently according to whether the threshold function $c$ is increasing or decreasing at that $t$. If decreasing, the banked ideas are irrelevant. Any banked idea that would be chosen at $t$ would also have been chosen at $t-d t$. If there is investment at $t$, it is because a viable idea materializes at that moment. On the other hand, if $c$ is increasing at $t$, then banked ideas may become viable. If $c$ is increasing, both the banked ideas and the increasing cost threshold affect the probability of investment at time $t$.

We will describe the probabilities of survival at each $t$ by reference to Figure 1, which shows an arbitrary cost threshold function. To describe the probability of investing in an 


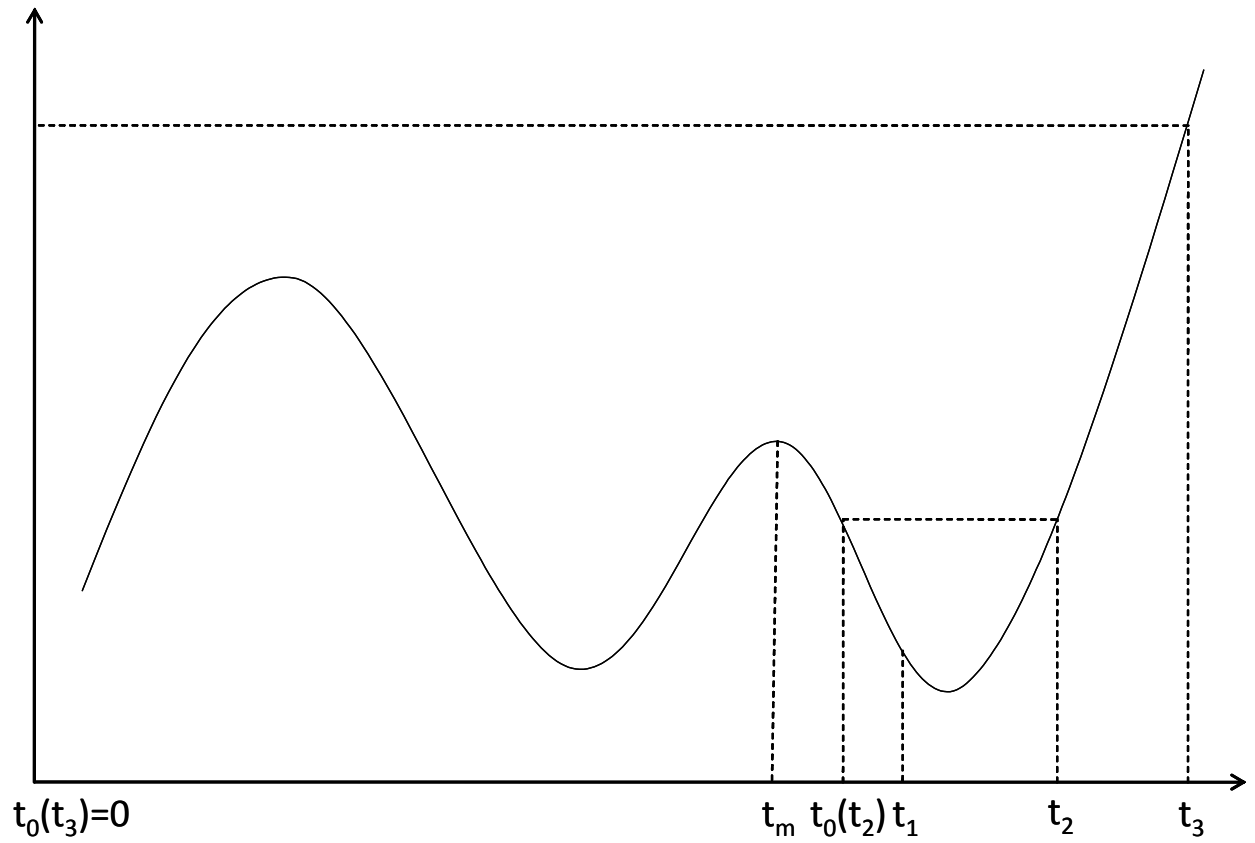

Figure 1: The stochastic process with banking

idea at any $t$ where $c$ is decreasing, such as $t_{1}$ in Figure 1 , let $t_{m}$ be the largest value smaller than $t$ where $c$ is nonincreasing. On the domain $\left[t_{m}, t\right]$, there are no viable banked ideas. Let $Q\left(t_{m} \mid \lambda, c\right)$ be the probability of surviving to $t_{m}$. Then the probability of survival to $t$, (that is, the probability that there is no viable idea by time $t$ ) is similar to the case without banking:

$$
Q\left(t_{m} \mid \lambda, c\right) e^{-\Lambda(t, c)+\Lambda\left(t_{m}, c\right)}
$$

where

$$
\Lambda(t, c)=\int_{0}^{t} \lambda F(c(\tilde{t})) d \tilde{t}
$$

This is the probability of survival to $t_{m}$ times the probability that no viable idea arrives in the interval $\left[t_{m}, t\right]$.

To describe the probabilities of survival at $t$ where $c$ is increasing, let $t_{0}(t)$ be the largest value smaller than $t$ such that $c\left(t_{0}(t)\right)=c(t)$. If there is no such value, let $t_{0}(t)=0$.

Two such points are $t_{2}$ and $t_{3}$ in Figure 1 . At $t_{2}$, the relevant banked ideas have been 
accumulating for a shorter period of time than at $t_{3}$. At $t_{2}$, any ideas below the cost threshold $c\left(t_{2}\right)$ that were received before $t_{0}\left(t_{2}\right)$ would have been used before $t_{0}\left(t_{2}\right)$. Therefore the relevant banked ideas are those which accumulated between $t_{0}\left(t_{2}\right)$ and $t_{2}$. At $t_{3}$, there may be relevant ideas with cost near $c\left(t_{3}\right)$ that accumulated very early, since there was never a time when such high-cost ideas were below the cost threshold.

The probability of survival to $t$ (that is, the probability that there is no viable idea by time $t$ ) is

$$
Q\left(t_{0}(t) \mid \lambda, c\right) e^{-\lambda F(c(t))\left[t-t_{0}(t)\right]}
$$

This is the probability of survival to $t_{0}(t)$ times the probability that no viable idea arrives in the interval $\left[t_{0}(t), t\right]$.

Thus, if ideas are banked, the stochastic process that determines the probability of survival until $t$ satisfies

$$
Q(t \mid \lambda, c)= \begin{cases}Q\left(t_{m} \mid \lambda, c\right) e^{-\Lambda(t, c)+\Lambda\left(t_{m}, c\right)} & \text { if } \quad c \text { is decreasing in }\left[t_{m}, t\right] \\ Q\left(t_{0}(t) \mid \lambda, c\right) e^{-\lambda F(c(t))\left[t-t_{0}(t)\right]} & \text { if } \quad c \text { is increasing at } t\end{cases}
$$

It is useful in the following analysis to define

$$
\mathcal{F}(t \mid c)= \begin{cases}F(c(t)) & \text { if } c^{\prime}(t) \leq 0 \\ {\left[F(c(t))+f(c(t))\left(t-t_{0}(t)\right) c^{\prime}(t)\right]} & \text { if } c^{\prime}(t)>0\end{cases}
$$

As seen from time $t$, the probability of arriving at $\hat{t}$ is $\frac{Q(\hat{t} \mid \lambda, c)}{Q(t \mid \lambda, c)}$. The probability that the first viable idea becomes available at $\hat{t}$ is the probability of arriving there, times the instantaneous probability that a viable idea arrives at time $\hat{t}$, namely,

$$
\frac{d}{d \hat{t}}\left[1-\frac{Q(\hat{t} \mid \lambda, c)}{Q(t \mid \lambda, c)}\right]=\lambda \mathcal{F}(\hat{t} \mid c) \frac{Q(\hat{t} \mid \lambda, c)}{Q(t \mid \lambda, c)}
$$

When $c$ is decreasing, the instantaneous probability of an innovation, $\lambda \mathcal{F}(\hat{t} \mid c)=\lambda F(c(\hat{t}))$, is the same as in the "use it or lose it" model, namely, the probability that a viable idea occurs in the interval $(\hat{t}, \hat{t}+d \hat{t})$. But when $c$ is increasing, the innovation may result from a banked idea rather than from an idea that occurs in the interval $(\hat{t}, \hat{t}+d \hat{t})$. The instantaneous probability of innovation is therefore larger, namely, $\lambda \mathcal{F}(\hat{t} \mid c)>\lambda F(c(\hat{t}))$. The 
instantaneous probability of innovation has two parts. First is the probability that a viable idea arrives to someone in $d \hat{t}$, namely $\lambda F(c(\hat{t})) d \hat{t}$. Second is the probability that a banked idea is called into play. When the threshold rises by $c^{\prime}(\hat{t}) d \hat{t}$, the probability that there is a banked idea in the cost band $c^{\prime}(\hat{t}) d \hat{t}$ is $\lambda f(c(\hat{t}))\left(\hat{t}-t_{0}(\hat{t})\right)$.

Conditional on surviving to time $t$, social welfare measured from time $t$ is $B$ defined by

$$
B(t, c, \lambda)=\int_{t}^{\infty} e^{-r(\hat{t}-t)}\left(\frac{v}{r}-E_{F}(c(\hat{t}))\right) \lambda \mathcal{F}(\hat{t} \mid c) \frac{Q(\hat{t} \mid \lambda, c)}{Q(t \mid \lambda, c)} d \hat{t}
$$

If the threshold function $c$ maximizes $B$, the following condition holds at each $t$.

$$
\left(\frac{v}{r}-c(t)\right)=B(t, c, \lambda)
$$

When ideas can be banked, it remains true that the welfare-maximizing cost threshold is stationary. If the threshold is stationary, then the probability distribution $Q(\cdot \mid \lambda, \bar{c})$ is the same as $P(\cdot \mid \lambda, \bar{c})$, so $\bar{B}(t, \bar{c}, \lambda)=\bar{V}(t, \bar{c}, \lambda)$. Thus, the first-order condition is the same as in the "use it or lose it" model, namely (7). Therefore, as recorded in the next proposition, the stationary cost thresholds are the same in both cases. ${ }^{3}$

Proposition 3 Suppose that the recipient of an idea can use it or bank it. Suppose that the arrival rate of ideas, $\lambda$, is fixed and known. Then, given $\lambda$, the welfare-maximizing cost threshold is stationary, has the same value $c^{*}(\lambda)$ as in the "use it or lose it" model, and is thus decreasing with $\lambda$.

We now turn to the case that ideas can be banked, and the hit rate of ideas, $\lambda$, is unknown. The prior is again $\tilde{h}$, and using the survival probabilities described in (12), the posterior distribution on $\lambda$ is again described by (8), substituting $Q$ for $P$. The analog to Lemma 1 holds for the distribution $Q$, by the same proof as for the distribution $P .{ }^{4}$ $E(\lambda \mid \hat{t}, c)$ decreases with $\hat{t}$.

\footnotetext{
${ }^{3}$ Banking is the same as recall in the search literature. See McCall and McCall (2008) for similar results in search theory with and without recall. As in the "use it or lose it" model, we give a different proof, using our social welfare function. The social welfare function is useful for understanding the case of unknown $\lambda$.

${ }^{4}$ Lemma 1 is proved by using Claim 2 in the proof. Claim 2 applies here because, for the distribution $Q$,

$$
\frac{d}{d t} h(\lambda \mid t, c)=\mathcal{F}(c(t)) h(\lambda \mid t, c)[E(\lambda \mid t, c)-\lambda] .
$$
}


We define $\tilde{Q}(\hat{t} \mid c)$ analogously to (9), except that the stochastic process underlying $Q(\hat{t} \mid \lambda, c)$ is defined by (12) instead of $(2)$. The social value of continuing from time $t$ is given by a function $\tilde{B}$, which we write in two ways. The first line is the definition, and the second line is equivalent, emphasizing that the belief on $\lambda$ is updated at each $\hat{t}$.

$$
\begin{aligned}
\tilde{B}(t, c, \tilde{h}) & =\int_{0}^{\infty} B(t, c, \lambda) h(\lambda \mid t, c) d \lambda \\
& =\int_{t}^{\infty} e^{-r(\hat{t}-t)}\left(\frac{v}{r}-E_{F}(c(\hat{t}))\right) E(\lambda \mid \hat{t}, c) \mathcal{F}(\hat{t} \mid c) \frac{\tilde{Q}(\hat{t} \mid c)}{\tilde{Q}(t \mid c)} d \hat{t}
\end{aligned}
$$

The welfare-maximizing $c$ again satisfies

$$
\frac{v}{r}-c(t)=\tilde{B}(t, c, \tilde{h})
$$

The following proposition shows that the welfare-maximizing cost threshold with banking is still increasing when the social planner is continuously updating his posterior about $\lambda$.

Proposition 4 Suppose that the recipient of an idea can use it or bank it. Suppose that the arrival rate of ideas, $\lambda$, has a prior distribution $\tilde{h}$ with support $[0, \infty)$. Let $c$ be the threshold function that maximizes $\tilde{B}(0, \cdot, \tilde{h})$. Then $c$ is increasing.

We conclude from Propositions 1 and 2, together with Propositions 3 and 4, that it is learning about $\lambda$ that causes the welfare-maximizing threshold to be increasing. It is not the banking of ideas per se.

Finally we show that optimized social welfare is higher with banking than without. When ideas are banked, the social planner is more pessimistic about $\lambda$ at each $t$ for a given $c$. At the same time, the arrival rate of viable ideas is higher when some of ideas may come from the idea bank. The next proposition shows that the latter effect dominates.

Proposition 5 Let $\mathcal{C}$ be the set of threshold functions $c$ that are increasing. Then for each $t>0, \max _{c \in \mathcal{C}} \tilde{B}(t, c, \tilde{h})>\max _{c \in \mathcal{C}} \tilde{V}(t, c, \tilde{h})$.

From (11) and (15), this proposition implies the following: 
Corollary 1 Let $c^{V}$ be the welfare-maximizing threshold function in the "use it or lose it" model, and let $c^{B}$ be the welfare-maximizing threshold function in the "use it or bank it" model. Then $c^{V}>c^{B}$.

The social planner prefers to be more selective when he can rely on banked ideas, even if he is more pessimistic about $\lambda$ at each $t$.

\section{Implementing the Optimal Cost Threshold}

The social planner cannot implement his preferred cost threshold directly, because the social planner is not the recipient of the ideas. Ideas for $R \& D$ are widely dispersed within the population of potential innovators. At best the social planner can try to implement the threshold by setting rewards.

We suppose that the social planner sets a reward function $\rho: \mathbf{R}_{+} \rightarrow \mathbf{R}_{+}$. For example, the reward function can represent patent policy or a prize system. The reward function $\rho$ implements the threshold function $c$ if the possessor of an idea with cost $c_{0}$ at time $t$ invests in the idea if and only if his idea satisfies $c_{0} \leq c(t)$. In the "use it or lose it" model, the only relevant ideas are those that just arrived, but in the "lose it or bank it" model, the relevant idea might previously have been banked.

We already pointed out how to implement the cost threshold in the "use it or lose it" model. We record it formally here.

Proposition 6 Suppose that $c$ is the welfare-maximizing cost threshold in the "use it or lose it" model. Then c can be implemented by setting $\rho(t)=c(t)$ for all $t$.

Implementation is also easy whenever the cost threshold is stationary. This occurs in both models when $\lambda$ is known:

Proposition 7 Let $c$ be the welfare-maximizing threshold function when the hit rate of ideas, $\lambda$, is fixed and known. Then $c$ is stationary, and can be implemented by a reward function that satisfies $\rho(t)=c(t)$ for all $t$. This applies in both the "use it or lose it" model 
and the "use it or bank it" model. In both cases, the welfare-maximizing stationary reward decreases with $\lambda$.

We interpret this proposition to mean that rewards should be higher when ideas are scarce.

Implementation is not as easy in the "use it or bank it" model when the hit rate of ideas is unknown. The intended cost threshold is increasing, which implies that the reward must be greater than the implemented cost threshold at each $t$. If equal, the recipient of a marginal idea (with cost equal to $c(t)$ ) would not invest as intended, since investing in the marginal idea would lead to zero profit. Since the reward function is increasing, the possessor of the marginal idea might make positive profit by waiting for some period until the reward is higher. Even if the possessor of the idea might be preempted during the delay, the expected profit with delay is still larger than zero. ${ }^{5}$

We suppose that the social planner chooses a reward function $\rho$, and the recipients of ideas choose investment strategies. Each recipient's investment strategy is a threshold function that indicates whether, when the opportunity arises, the possessor of the idea will invest in the idea or bank it. The planner's objective is to make sure that the privately chosen threshold functions correspond to the threshold function that maximizes welfare as a whole.

Each idea recipient's incentive to bank or invest depends on his belief about $\lambda$, and also on his belief about the other agents' investment strategies. If there is a large accumulation of banked ideas, the probability of being preempted is high.

Further, the social planner must predict these beliefs. If the social planner does not know the beliefs of the idea recipients, he cannot predict their investment strategies, and thus cannot predict the cost threshold that will be implemented by his reward function.

We solve the problem of beliefs in a familiar way. We require that beliefs must be correct in equilibrium. For the threshold function $c$ that will be implemented, a recipient

\footnotetext{
${ }^{5}$ A similar type of tradeoff exists in Weeds (2002), who considers a model of R\&D competition where delay is undermined by the fear of pre-emption.
} 
must believe in equilibrium that other agents invest according to $c$, and the recipient must find it most profitable to invest according to $c$ himself. The point is to find a reward function with this result.

The planner's belief about $\lambda$ is irrelevant in the following discussion. The planner can implement any nondecreasing threshold he wants, provided he knows the beliefs of the idea recipients. The idea recipients have more information than the planner, and will have different beliefs than the planner. This is because, when a nonviable idea arrives to a recipient, the arrival contains information about $\lambda$ even if the idea is banked instead of used.

Let $\hat{h}(\cdot \mid t, c)$ represent the belief of each recipient about $\lambda$, with expected value $\hat{E}(\lambda \mid t, c)$. The argument $c$ is a belief, namely, the recipient's belief about the investment strategy (cost threshold) of the other recipients. Our notation $\hat{h}$ incorporates an assumption about equilibrium: that in equilibrium all other recipients of ideas obey the same investment strategy, $c$. We justify this assumption after defining the idea recipients' profit function.

A recipient's belief $\mathcal{P}$ on the probability of arriving at $\hat{t}$ is given by

$$
\mathcal{P}(\hat{t} \mid c)=\int_{0}^{\infty} Q(\hat{t} \mid \lambda, c) \tilde{h}(\lambda) d \lambda
$$

where $c$ is the threshold function that other recipients are assumed to obey, and $\hat{h}$ is the recipients' posterior belief on $\lambda$. The probability of arriving at $\hat{t}$, having already arrived at $t$, is given by

$$
\frac{\mathcal{P}(\hat{t} \mid c)}{\mathcal{P}(t \mid c)}=\int_{0}^{\infty} \frac{Q(\hat{t} \mid \lambda, c)}{Q(t \mid \lambda, c)} \hat{h}(\lambda \mid t, c) d \lambda
$$

To define the idea recipient's profit function, suppose that he possesses an idea with cost $c_{0}$ at time $t$. The innovator's profit, as a function of the time $\hat{t}$ at which he will invest, is given by (16).

$$
\pi\left(\hat{t}, c_{0} \mid t, c\right)=\left(\rho(\hat{t})-c_{0}\right) e^{-r(\hat{t}-t)} \frac{\mathcal{P}(\hat{t} \mid c)}{\mathcal{P}(t \mid c)}
$$


The derivative of the profit function is

$$
\begin{aligned}
\frac{d}{d \hat{t}} \pi\left(\hat{t}, c_{0} \mid t, c\right) & =\rho^{\prime}(\hat{t}) e^{-r(\hat{t}-t)} \frac{\mathcal{P}(\hat{t} \mid c)}{\mathcal{P}(t \mid c)}-\left(\rho(\hat{t})-c_{0}\right) e^{-r(\hat{t}-t)}\left[r \frac{\mathcal{P}(\hat{t} \mid c)}{\mathcal{P}(t \mid c)}+\frac{d}{d \hat{t}} \frac{\mathcal{P}(\hat{t} \mid c)}{\mathcal{P}(t \mid c)}\right] \\
& =e^{-r(\hat{t}-t)} \frac{\mathcal{P}(\hat{t} \mid c)}{\mathcal{P}(t \mid c)} \times\left(\rho^{\prime}(\hat{t})-\left(\rho(\hat{t})-c_{0}\right)[r+\mathcal{F}(\hat{t} \mid c) \hat{E}(\lambda \mid \hat{t}, c)]\right)
\end{aligned}
$$

where

$$
\hat{E}(\lambda \mid \hat{t}, c)=\int_{0}^{\infty} \lambda \hat{h}(\lambda \mid \hat{t}, c) d \lambda
$$

The profit-maximizing investment decision at $\hat{t}$ is

$$
\begin{aligned}
\text { delay if } \rho^{\prime}(\hat{t}) & >(r+\hat{E}(\lambda \mid \hat{t}, c) \mathcal{F}(\hat{t} \mid c))\left[\rho(\hat{t})-c_{0}\right] \\
\text { invest if } \rho^{\prime}(\hat{t}) & \leq(r+\hat{E}(\lambda \mid \hat{t}, c) \mathcal{F}(\hat{t} \mid c))\left[\rho(\hat{t})-c_{0}\right]
\end{aligned}
$$

In (17), $\rho^{\prime}$ on the left hand side is the benefit of delay. The right hand side is the cost of delay, namely, the interest cost $r$ on the foregone profit $\rho(\hat{t})-c_{0}$, and the perceived probability $\hat{E}(\lambda \mid \hat{t}, c) \mathcal{F}(\hat{t} \mid c)$ of being preempted.

The investment strategy derived in (17) is the same for all idea recipients. However, the derivation is based on the prior assumption that all idea recipients have the same belief about $\lambda$. This is justified in the following Remark.

We write the following as a remark instead of a lemma because the proof in the appendix elaborates the model, assuming that the population of idea recipients is finite instead of infinite, and taking limits. We do this in order to derive limit beliefs as the population becomes large. We show that, in the limit, the beliefs of idea recipients do not depend on when a recipient received his idea. In the limit, the probability of receiving an idea is zero, and the timing of the idea has negligible impact beyond the impact of receiving one. Nevertheless, the limit beliefs are more optimistic than those of the planner because the planner has not observed the arrival of any idea at all.

Remark 1 Let c be an arbitrary nondecreasing investment strategy (cost threshold). (a) If all recipients of ideas believe that $c$ is the investment strategy of every other recipient, then at a given time $t$, every recipient of an idea has the same belief on arrival rates, which we 
call $\hat{h}(\cdot \mid t, c)$. (b) The recipients' belief $\hat{h}(\cdot \mid t, c)$ stochastically dominates the planner's belief $h(\cdot \mid t, c)$ at every $t$, and as a consequence, $\hat{E}(\lambda \mid t, c)>E(\lambda \mid t, c) .(c)$ Every recipient of an idea with a given cost, say $c_{0}$, has the same profit-maximizing investment strategy given by $(17)$.

The investment behavior (17) should guide the planner in choosing his reward function. For an arbitrary nondecreasing cost threshold $c$, let the reward function satisfy

$$
\rho^{\prime}(t)=(r+\hat{E}(\lambda \mid t, c) \mathcal{F}(t \mid c))[\rho(t)-c(t)]
$$

The following lemma says that, with the reward function defined in (18), idea recipients will indeed obey the threshold function $c$ that determines beliefs (represented by $\hat{E}(\lambda \mid t, c) \mathcal{F}(t \mid c))$.

Lemma 2 Let $c$ be a nondecreasing cost threshold, and suppose that the belief of each idea recipient is c. Suppose that the reward function $\rho$ solves (18) at every $t$. Then for each $t$, a recipient's most profitable investment strategy is to invest if he has an idea with cost $c_{0} \leq c(t)$, and not otherwise.

Proof: The condition (17) is clearly profit-maximizing at the margin, for choosing whether to invest at $t$ or delay for a length of time $d t$. We must also show that if (17) holds, a longer delay is also not profitable.

$$
\begin{aligned}
& \text { If } c_{0} \leq c(t) \leq c(\tilde{t}) \text {, then } \\
& \qquad \rho^{\prime}(t)=(r+\hat{E}(\lambda \mid t, c) \mathcal{F}(t \mid c))[\rho(t)-c(t)] \leq(r+\hat{E}(\lambda \mid t, c) \mathcal{F}(t \mid c))\left[\rho(t)-c_{0}\right]
\end{aligned}
$$

and

$$
\rho^{\prime}(\tilde{t})=(r+\hat{E}(\lambda \mid \tilde{t}, c) \mathcal{F}(\tilde{t} \mid c))[\rho(\tilde{t})-c(\tilde{t})] \leq(r+\hat{E}(\lambda \mid \tilde{t}, c) \mathcal{F}(\tilde{t} \mid c))\left[\rho(\tilde{t})-c_{0}\right]
$$

The first line (respectively, second line) means that it is more profitable to invest at $t$ rather than $t+d t$ (respectively, $\tilde{t}$ rather than $\tilde{t}+d t$ ) because the additional profit from delay (the left hand term) is no greater than the cost of delay (the right hand term). The first 
line (respectively, second line) holds because $c_{0} \leq c(t)$ (respectively, $c_{0} \leq c(\tilde{t})$ ). If the idea was not available at $t$ (or if the possessor of the idea made a mistake by not investing), he will invest at the earliest next time, such as at $\tilde{t}$, since $c_{0} \leq c(t)$ implies $c_{0} \leq c(\tilde{t})$ whenever $t<\tilde{t}$. At any time after $t$, the possessor of an idea with cost less than $c(t)$ prefers to invest rather than bank.

Thus, if $c$ is nondecreasing and the reward function $\rho$ is chosen to satisfy (18), recipients of ideas will invest according to the investment strategy $c$. We therefore say that $\rho$ implements $c$ if $\rho$ satisfies (18) and also satisfies $\rho(t) \geq c(t)$.

Proposition 8 Suppose that $c$ is nondecreasing. There exists a reward function $\rho$ that implements $c$. The function $\rho$ satisfies $\rho>c$ if $c$ is increasing. Further, $\rho(t)-c(t) \rightarrow 0$ as $t \rightarrow \infty$.

Proof: Let

$$
\begin{aligned}
\rho(t) & =c(t)+\frac{k-\int_{0}^{t} e^{-D(\hat{t})} c^{\prime}(\hat{t}) d \hat{t}}{e^{-D(t)}} \\
\text { where } D(\hat{t}) & =\int_{0}^{\hat{t}}(r+\hat{E}(\lambda \mid \tilde{t}, c) \mathcal{F}(\tilde{t} \mid c)) d \tilde{t} \\
k & =\lim _{t \rightarrow \infty} \int_{0}^{t} e^{-D(\hat{t})} c^{\prime}(\hat{t}) d \hat{t} .
\end{aligned}
$$

The function $\rho$ defined by (19) is a solution to (18). Since $\int_{0}^{t} e^{-D(\hat{t})} c^{\prime}(\hat{t}) d \hat{t}$ is nondecreasing with $t$, the choice of $k$ ensures that $\rho(t)-c(t) \geq 0$ for all $t$, with strict inequality if $c$ is increasing on some domain. The definition of $k$ can be satisfied because at each $t$, $\int_{0}^{t} e^{-D(\hat{t})} c^{\prime}(\hat{t}) d \hat{t} \leq \int_{0}^{t} c^{\prime}(\hat{t}) d \hat{t}=c(t)-c(0)$. Since $c$ is nondecreasing and bounded by $(v / r)$, there exists $\bar{c} \leq(v / r)$ such that $c(t) \rightarrow \bar{c}$.

It holds that $\rho(t)-c(t) \rightarrow 0$ because

$$
\frac{k-\int_{0}^{t} e^{-D(\hat{t})} c^{\prime}(\hat{t}) d \hat{t}}{e^{-D(t)}} \rightarrow 0
$$

by L'Hopital's rule:

$$
\frac{e^{-D(t)} c^{\prime}(t)}{e^{-D(t)}(r+\hat{E}(\lambda \mid t, c) \mathcal{F}(t \mid c))}=\frac{c^{\prime}(t)}{(r+\hat{E}(\lambda \mid t, c) \mathcal{F}(t \mid c))} \rightarrow 0
$$


We close this section with a comment on the profitability of $R \& D$ in aggregate. Due to the scarcity of ideas, innovators make positive profit on average. This is because the cost of an implemented idea will generally be lower than the threshold. The recipient of a low-cost idea is in a favored position, and everyone would like to have such an idea, but there is little that one can do to create the investment opportunity. In fact, we have taken the extreme assumption that investment opportunities arrive entirely by chance. We have done this to emphasize our key departure from the more standard R\&D literature, where all firms have access to an investment opportunity, and profit may be dissipated in a patent race or through preemptive strategies. As a consequence, one would not expect to observe in equilibrium that the return to $R \& D$ investments is the same as the return to capital. On average, it should be higher.

Our argument seems to overlook the possibility that an innovator might be able to keep his innovation secret while charging a proprietary price. This would presumably subvert the objective of the reward, and possibly not implement the intended cost threshold. However, as we show in our (2007) paper, at least in the case of known $\lambda$, secrecy is never preferred by the recipient of an idea. ${ }^{6}$ This assumes that with secrecy, another innovator can claim the reward and end the prior innovator's proprietary profit stream.

\section{Economic Concepts and Legal Concepts}

We interpret ideas, and the fact that ideas are private, as a model of imagination or creativity. Ideas have economic value because they are scarce. We have argued that rewards should be higher in environments where ideas are scarce. If ideas are scarce, higher cost should be tolerated in order to reduce delay. We have also argued that rewards should be increasing as time passes without an innovation. Longer delay leads to expectation of an even longer delay. The delay can be mitigated with higher rewards, since higher rewards encourage investment in higher-cost ideas. Because ideas are not common knowledge, innovators make

\footnotetext{
${ }^{6}$ This result contrasts with previous treatments of secrecy in the literature. See, for example, Denicolo and Franzoni (2004) and Erkal (2005).
} 
positive profit in expectation.

These arguments apply equally well to patents and prizes, and any other way of giving rewards. ${ }^{7}$ Patents raise the issue of whether our prescriptions can be implemented under existing patent doctrine. They also raise the question of how deadweight loss incurred in collecting the reward money changes the cost threshold that society might want to implement.

The main requirements for obtaining a patent are novelty, nonobviousness, utility and enablement. Together, these requirements govern whether a patent is granted and the breadth of the claims that are granted. To maximize social welfare as defined in this paper, patent grants would be more generous when ideas are scarce, or more particularly, when the innovation arrives after long delay. Two ways of being generous are, first, to grant the patent, and second, to grant broad claims. For the granting of a patent, a relevant doctrine is the nonobviousness requirement. One of the secondary considerations for patentability is "long-felt need," and it seems to correspond quite closely to the problem of delay that we have described.

\section{Appendix}

\subsection{Proof of Proposition 1}

(a) We first show that the optimized value of $V$ is stationary. Stationarity of $c$ follows from $(5) \cdot{ }^{8}$

Claim 1 Given $t_{1}<t_{2}$, let $c_{1}:\left(t_{1}, \infty\right) \rightarrow \mathbf{R}_{+}$be the function that maximizes $V\left(t_{1}, c, \lambda\right)$, and let $c_{2}:\left(t_{2}, \infty\right) \rightarrow \mathbf{R}_{+}$be the function that maximizes $V\left(t_{2}, c, \lambda\right)$. Then $V\left(t_{1}, c_{1}, \lambda\right)=$ $V\left(t_{2}, c_{2}, \lambda\right)$.

Proof: Define a function $\tilde{c}_{1}:\left(t_{1}, \infty\right) \rightarrow \mathbf{R}_{+}$by

$$
\tilde{c}_{1}(\hat{t})=c_{2}\left(\hat{t}+t_{2}-t_{1}\right)
$$

\footnotetext{
${ }^{7}$ For a sample of the many ways, other than patents, that economists have thought about incentives in R\&D, see Wright (1983), chapters 2 and 8 of Scotchmer (2004) and Hopenhayn, Llobet and Mitchell (2006).

${ }^{8}$ Stationarity is proved in the search literature by using a value function and the Bellman equation. We take a different approach because $V$ is useful when we discuss the social welfare function $\tilde{V}$ for unknown $\lambda$.
} 
The function $\tilde{c}_{1}$ is the same function as $c_{2}$, except shifted to begin at $t_{1}$ instead of $t_{2}$. Then by definition, $V\left(t_{1}, c_{1}, \lambda\right) \geq V\left(t_{1}, \tilde{c}_{1}, \lambda\right)$, and by construction, $V\left(t_{1}, \tilde{c}_{1}, \lambda\right)=V\left(t_{2}, c_{2}, \lambda\right)$. Hence, $V\left(t_{1}, c_{1}, \lambda\right) \geq V\left(t_{2}, c_{2}, \lambda\right)$.

Now reverse the roles and define a threshold function $\tilde{c}_{2}:\left(t_{2}, \infty\right) \rightarrow \mathbf{R}_{+}$by

$$
\tilde{c}_{2}(\hat{t})=c_{1}\left(\hat{t}-t_{2}+t_{1}\right)
$$

Then by definition, $V\left(t_{2}, c_{2}, \lambda\right) \geq V\left(t_{2}, \tilde{c}_{2}, \lambda\right)$, and by construction, $V\left(t_{2}, \tilde{c}_{2}, \lambda\right)=V\left(t_{1}, c_{1}, \lambda\right)$. Hence, $V\left(t_{2}, c_{2}, \lambda\right) \geq V\left(t_{1}, c_{1}, \lambda\right)$. Together with $V\left(t_{1}, c_{1}, \lambda\right) \geq V\left(t_{2}, c_{2}, \lambda\right)$, this proves the result.

Claim 1 implies that $V$ has a constant value. Using (5), this implies that $c$ is also stationary.

Part (b) follows by differentiating (7) implicitly.

\subsection{Proof of Lemma 1}

The lemma follows from Claim 2. When the stochastic process is given by (2) as in section 3 , and $h$ is given by (8), the hypothesis of the Claim is satisfied because

$$
\frac{d}{d t} h(\lambda \mid t, c)=F(c(t)) h(\lambda \mid t, c)[E(\lambda \mid t, c)-\lambda]
$$

Claim 2 Suppose there exists $\hat{\lambda}$ such that $\frac{d}{d t} h(\lambda \mid t, c)>0$ for $\lambda<\hat{\lambda}$ and $\frac{d}{d t} h(\lambda \mid t, c)<0$ for $\lambda>\hat{\lambda}$. Then $\frac{d}{d t} H(\lambda \mid t, c)>0$ at each $\lambda \in[0, \infty)$.

Proof: For each $\lambda \in[0, \infty)$,

$$
0=\frac{d}{d t} \int_{0}^{\infty} h(\tilde{\lambda} \mid t, c) d \tilde{\lambda}=\frac{d}{d t} \int_{0}^{\lambda} h(\tilde{\lambda} \mid t, c) d \tilde{\lambda}+\frac{d}{d t} \int_{\lambda}^{\infty} h(\tilde{\lambda} \mid t, c) d \tilde{\lambda}
$$

For $\lambda \leq \hat{\lambda}$

$$
\frac{d}{d t} H(\lambda \mid t, c)=\int_{0}^{\lambda} \frac{d}{d t} h(\tilde{\lambda} \mid t, c) d \tilde{\lambda}>0
$$

For $\lambda>\hat{\lambda}$,

$$
\begin{aligned}
\frac{d}{d t} H(\lambda \mid t, c) & =\int_{0}^{\lambda} \frac{d}{d t} h(\tilde{\lambda} \mid t, c) d \tilde{\lambda}=\int_{0}^{\infty} \frac{d}{d t} h(\tilde{\lambda} \mid t, c) d \tilde{\lambda}-\int_{\lambda}^{\infty} \frac{d}{d t} h(\tilde{\lambda} \mid t, c) d \tilde{\lambda} \\
& =0-\int_{\lambda}^{\infty} \frac{d}{d t} h(\tilde{\lambda} \mid t, c) d \tilde{\lambda}>0
\end{aligned}
$$

Therefore, $H\left(\cdot \mid t_{1}, c\right)$ stochastically dominates $H\left(\cdot \mid t_{2}, c\right)$ and $(d / d t) E(\lambda \mid t, c)<0$. 


\subsection{Proof of Proposition 2}

The conclusion that $c$ is increasing follows from (11) since we can show that $\tilde{V}$ is decreasing.

For the derivative of $\tilde{V}$, we need the derivative of the conditional density function at $\hat{t}>t$,

$$
\frac{d}{d t} E(\lambda \mid \hat{t}, c) F(c(\hat{t})) \frac{\tilde{P}(\hat{t} \mid c)}{\tilde{P}(t \mid c)}=F(c(t)) E(\lambda \mid t, c) \gamma(\hat{t} \mid t, c, \lambda) h(\lambda \mid t, c)
$$

Differentiating $\tilde{V}$ with respect to $t$ gives

$$
\begin{aligned}
& \frac{d}{d t} \tilde{V}(t, c, \tilde{h}) \\
= & -\left(\frac{v}{r}-E_{F}(c(t))\right) E(\lambda \mid t, c) F(c(t))+r \tilde{V}(t, c, \tilde{h}) \\
& +\int_{t}^{\infty} e^{-r(\hat{t}-t)}\left(\frac{v}{r}-E_{F}(c(\hat{t}))\right) E(\lambda \mid \hat{t}, c) F(c(\hat{t})) \frac{d}{d t}\left[\frac{\tilde{P}(\hat{t} \mid c)}{\tilde{P}(t \mid c)}\right] d \hat{t} \\
= & -\left(\frac{v}{r}-E_{F}(c(t))\right) E(\lambda \mid t, c) F(c(t))+r \tilde{V}(t, c, \tilde{h}) \\
& +E(\lambda \mid t, c) F(c(t)) \int_{t}^{\infty} e^{-r(\hat{t}-t)}\left(\frac{v}{r}-E_{F}(c(\hat{t}))\right) E(\lambda \mid \hat{t}, c) F(c(\hat{t})) \frac{\tilde{P}(\hat{t} \mid c)}{\tilde{P}(t \mid c)} d \hat{t} \\
= & -\left(\frac{v}{r}-E_{F}(c(t))\right) E(\lambda \mid t, c) F(c(t))+(r+E(\lambda \mid t, c) F(c(t))) \tilde{V}(t, c, \tilde{h}) \\
= & (r+E(\lambda \mid t, c) F(c(t)))\left[-\left(\frac{v}{r}-E_{F}(c(t))\right) \frac{E(\lambda \mid t, c) F(c(t))}{r+E(\lambda \mid t, c) F(c(t))}+\tilde{V}(t, c, \tilde{h})\right] \\
= & (r+E(\lambda \mid t, c) F(c(t)))\left[-\left(\frac{v}{r}-E_{F}(c(t))\right) \frac{E(\lambda \mid t, c) F(c(t))}{(r+E(\lambda \mid t, c)) F(c(t))}+\left(\frac{v}{r}-c(t)\right)\right]
\end{aligned}
$$

where the last line follows from (11).

First, the optimizing function $c$ cannot be "U-shaped" on any domain. If the function $c$ is "U-shaped" on some domain, there exist $t_{1}$ and $t_{2}$ such that $t_{1}<t_{2}, c\left(t_{1}\right)=$ $c\left(t_{2}\right)$, and $c^{\prime}\left(t_{1}\right)<0<c^{\prime}\left(t_{2}\right)$. However, this generates a contradiction. It holds that $\left(\frac{v}{r}-E_{F}\left(c\left(t_{1}\right)\right)\right)=\left(\frac{v}{r}-E_{F}\left(c\left(t_{2}\right)\right)\right),\left(\frac{v}{r}-c\left(t_{1}\right)\right)=\left(\frac{v}{r}-c\left(t_{1}\right)\right), F\left(c\left(t_{1}\right)\right)=F\left(c\left(t_{2}\right)\right)$, and (using Lemma 1) $E\left(\lambda \mid t_{1}, c\right)>E\left(\lambda \mid t_{2}, c\right)$. Hence, using $(22), \frac{d}{d t} \tilde{V}\left(t_{1}, c, \tilde{h}\right)<\frac{d}{d t} \tilde{V}\left(t_{2}, c, \tilde{h}\right)$. Together with $c^{\prime}\left(t_{1}\right)<0<c^{\prime}\left(t_{2}\right)$, this contradicts (11).

Proposition 2 then follows from Claim 3 and Claim 4 below. By Claim 4, if $c$ maximizes $\tilde{V}$, then $\tilde{V}(t, c, \tilde{h})$ is decreasing with $t$ on a domain $(\bar{t}, \infty)$. Therefore, using (11), it also holds that $c$ is increasing on that domain. But it then follows that the entire function $c$ 
is nondecreasing, since $c$ cannot be U-shaped on any domain. And, in fact, $c$ is increasing because the derivative (22) is not constant on any interval.

Claim 3 Let $c$ be the threshold function that maximizes $V(0, \cdot, \tilde{h})$. Then there exists $\bar{t}$ such that the function $t \rightarrow e^{-r t}\left(\frac{v}{r}-E_{F}(c(t))\right)$ is decreasing on the domain $(\bar{t}, \infty)$.

Proof of Claim 3: Because the optimal c cannot be U-shaped, it is either nonincreasing or nondecreasing for sufficiently large $t$. Further, because $c$ is bounded above and below, it holds that $c^{\prime}(t) \rightarrow 0, c(t) \rightarrow c^{*}, E_{F}(c(t)) \rightarrow E_{F}\left(c^{*}\right)$ for some $c^{*} \in\left[0, \frac{v}{r}\right]$. The result follows because

$$
\begin{aligned}
\frac{d}{d t} e^{-r t}\left(\frac{v}{r}-E_{F}(c(t))\right) & =e^{-r t}\left[-r\left(\frac{v}{r}-E_{F}(c(t))\right)-\frac{d E_{F}(c(t))}{d c(t)} c^{\prime}(t)\right] \\
& \rightarrow \quad-r e^{-r t}\left(\frac{v}{r}-E_{F}\left(c^{*}\right)\right)
\end{aligned}
$$

Claim 4 Let $c$ be the threshold function that maximizes $V(0, \cdot, \tilde{h})$. Then there exists a domain $(\bar{t}, \infty)$ for which

$$
\tilde{V}\left(t_{1}, c, \tilde{h}\right)>\tilde{V}\left(t_{2}, c, \tilde{h}\right) \quad \text { if } \quad \bar{t} \leq t_{1}<t_{2}
$$

Proof of Claim 4: We will take the domain $(\bar{t}, \infty)$ as the domain on which $e^{-r t}\left(\frac{v}{r}-E_{F}(c(t))\right)$ is decreasing, by Claim 3. We will show that

$$
\tilde{V}\left(t_{1}, c, \tilde{h}\right) \geq \tilde{V}\left(t_{1}, \tilde{c}, \tilde{h}\right)>\tilde{V}\left(t_{2}, c, \tilde{h}\right) \text { if } \bar{t} \leq t_{1}<t_{2}
$$

where $\tilde{c}$ is defined by $\tilde{c}(t)=c(t)$ for $t \leq t_{1}$ and $\tilde{c}(t)=c\left(t+t_{2}-t_{1}\right)$ for $t>t_{1}$.

The first inequality in (23) is true by the principle of optimality. Beginning from time $t_{1}$, the optimizing function is still $c$, as it was when optimized from the beginning. If $\bar{c}$ satisfies $\tilde{V}\left(t_{1}, \bar{c}, \tilde{h}\right) \geq \tilde{V}\left(t_{1}, \hat{c}, \tilde{h}\right)$ for all threshold functions $\hat{c}$, then $\bar{c}(t)=c(t)$ for every $t \geq t_{1}$

It is the second inequality in (23) that we must show. The function $\tilde{c}$ in $\tilde{V}\left(t_{1}, \tilde{c}, \tilde{h}\right)$ is defined by the function $c$ restricted to $\left(t_{2}, \infty\right)$ and shifted back in time to $t_{1}$. For a fixed 
$\lambda$, it therefore holds that $V\left(t_{1}, \tilde{c}, \lambda\right)=V\left(t_{2}, c, \lambda\right)$. Further, $h\left(\lambda \mid t_{1}, \tilde{c}\right)=h\left(\lambda \mid t_{1}, c\right)$ because $c=\tilde{c}$ for $t \leq t_{1}$. Therefore,

$$
\tilde{V}\left(t_{1}, \tilde{c}, \tilde{h}\right)=\int_{0}^{\infty} V\left(t_{1}, \tilde{c}, \lambda\right) h\left(\lambda \mid t_{1}, \tilde{c}\right) d \lambda=\int_{0}^{\infty} V\left(t_{2}, c, \lambda\right) h\left(\lambda \mid t_{1}, c\right) d \lambda,
$$

and to show the second inequality in (23), it is enough to show that

$$
\int_{0}^{\infty} V\left(t_{2}, c, \lambda\right) h\left(\lambda \mid t_{1}, c\right) d \lambda>\int_{0}^{\infty} V\left(t_{2}, c, \lambda\right) h\left(\lambda \mid t_{2}, c\right) d \lambda=\tilde{V}\left(t_{2}, c, \tilde{h}\right) .
$$

Since $e^{-r\left(t-t_{2}\right)}\left(\frac{v}{r}-E_{F}(c(t))\right)$ is decreasing with $t$ for $t \in(\bar{t}, \infty), V\left(t_{2}, c, \cdot\right)$ increases with $\lambda$. Then (24) follows because, by Lemma 1 , the distribution $h\left(\cdot \mid t_{1}, c\right)$ stochastically dominates $h\left(\cdot \mid t_{2}, c\right)$. This means that $h\left(\cdot \mid t_{2}, c\right)$ puts relatively more weight on low values of $\lambda$, where the value of $V\left(t_{2}, c, \lambda\right)$ is low, and $h\left(\cdot \mid t_{1}, c\right)$ puts relatively more weight on high values of $\lambda$, where the value of $V\left(t_{2}, c, \lambda\right)$ is high.

\subsection{Proof of Proposition 3}

We show this in two claims.

Claim 5 Let $c:\left(t_{1}, \infty\right) \rightarrow \mathbf{R}_{+}$be the function that maximizes $B(0, c, \lambda)$. Then $c$ is not increasing on any interval.

Proof: Suppose to the contrary that $c$ is increasing on a domain $\left[0, t_{2}\right]$. (The same argument works for any domain where $c$ is increasing.) Define a threshold function $\tilde{c}$ : $(0, \infty) \rightarrow \mathbf{R}_{+}$by

$$
\tilde{c}(\hat{t})= \begin{cases}c(\hat{t}) & \text { for } \quad \hat{t} \leq t_{2} \\ c\left(\hat{t}-t_{2}\right) & \text { for } \quad \hat{t}>t_{2}\end{cases}
$$

Thus, the function $\tilde{c}$ is identical to the optimizing function $c$ until $t_{2}$, but then the function $c$ repeats, so that $c(0)=\tilde{c}\left(t_{2}\right)$. During the period $\left[0, t_{2}\right]$, ideas are being banked. When the threshold function is $\tilde{c}$, the banked ideas may be useful at times $\tilde{t}>2 t_{2}$, when it holds that $\tilde{c}(t)>c\left(t_{2}\right)=\tilde{c}\left(2 t_{2}\right)$.

We will show that $B\left(t_{2}, c, \lambda\right)<B(0, c, \lambda)<B\left(t_{2}, \tilde{c}, \lambda\right)$, which contradicts the fact that $c$ is optimal from time $t_{2}$. Using (13), the first inequality holds because $c$ is assumed optimal 
and increasing, so $B$ is decreasing. We show the second inequality.

$$
\begin{aligned}
& B\left(t_{2}, \tilde{c}, \lambda\right)=\int_{t_{2}}^{2 t_{2}} e^{-r\left(t-t_{2}\right)}\left\{\begin{array}{l}
\left(\frac{v}{r}-E_{F}(\tilde{c}(t))\right) \times \\
\lambda\left[F(\tilde{c}(t))+f(\tilde{c}(t)) \tilde{c}^{\prime}(t)\left(t-t_{2}\right)\right] \times \\
e^{-\lambda F(\tilde{c}(t))\left(t-t_{2}\right)}
\end{array}\right\} d t \\
& +\int_{2 t_{2}}^{\infty} e^{-r\left(t-t_{2}\right)}\left\{\begin{array}{l}
\left(\frac{v}{r}-E_{F}(\tilde{c}(t))\right) \times \\
\lambda\left[F(\tilde{c}(t))+f(\tilde{c}(t)) \tilde{c}^{\prime}(t) t\right] \times \\
e^{-\lambda F(\tilde{c}(t))\left(t-t_{2}\right)}
\end{array}\right\} d t \\
& =\int_{t_{2}}^{2 t_{2}} e^{-r\left(t-t_{2}\right)}\left\{\begin{array}{l}
\left(\frac{v}{r}-E_{F}\left(c\left(t-t_{2}\right)\right)\right) \times \\
\lambda\left[F\left(c\left(t-t_{2}\right)\right)+f\left(c\left(t-t_{2}\right)\right) c^{\prime}\left(t-t_{2}\right)\left(t-t_{2}\right)\right] \times \\
e^{-\lambda F\left(c\left(t-t_{2}\right)\right)\left(t-t_{2}\right)}
\end{array}\right\} d t \\
& +\int_{2 t_{2}}^{\infty} e^{-r\left(t-t_{2}\right)}\left\{\begin{array}{l}
\left(\frac{v}{r}-E_{F}\left(c\left(t-t_{2}\right)\right)\right) \times \\
\lambda\left[F\left(c\left(t-t_{2}\right)\right)+f\left(c\left(t-t_{2}\right)\right) c^{\prime}\left(t-t_{2}\right) t\right] \times \\
e^{-\lambda F\left(c\left(t-t_{2}\right)\right)\left(t-t_{2}\right)}
\end{array}\right\} d t \\
& =\int_{0}^{t_{2}} e^{-r \hat{t}}\left\{\begin{array}{l}
\left(\frac{v}{r}-E_{F}(c(\hat{t}))\right) \times \\
\lambda\left[F(c(\hat{t}))+f(c(\hat{t})) c^{\prime}(\hat{t}) \hat{t}\right] \times \\
e^{-\lambda F(c(\hat{t})) \hat{t}}
\end{array}\right\} d \hat{t} \\
& +\int_{t_{2}}^{\infty} e^{-r \hat{t}}\left\{\begin{array}{l}
\left(\frac{v}{r}-E_{F}(c(\hat{t}))\right) \times \\
\lambda\left[F(c(\hat{t}))+f(c(\hat{t})) c^{\prime}(\hat{t})\left(\hat{t}+t_{2}\right)\right] \times \\
e^{-\lambda F(c(\hat{t}))}
\end{array}\right\} d \hat{t} \\
& =\int_{0}^{\infty}\left(\frac{v}{r}-E_{F}(c(t))\right) \tilde{G}^{\prime}\left(t \mid t_{2}\right) d t
\end{aligned}
$$

where

$$
\tilde{G}^{\prime}\left(\hat{t} \mid t_{2}\right)=\left\{\begin{array}{ll}
\lambda\left[F(c(\hat{t}))+f(c(\hat{t})) c^{\prime}(\hat{t}) \hat{t}\right] e^{-\lambda F(c(\hat{t})) \hat{t}} & \text { if } \quad \hat{t} \in\left[0, t_{2}\right] \\
\lambda\left[F(c(\hat{t}))+f(c(\hat{t})) c^{\prime}(\hat{t})\left(\hat{t}+t_{2}\right)\right] e^{-\lambda F(c(\hat{t})) \hat{t}} & \text { if } \quad \hat{t} \in\left[t_{2}, \infty\right]
\end{array}\right\}
$$

Similarly, write

$$
B(0, c, \lambda)=\int_{0}^{\infty}\left(\frac{v}{r}-E_{F}(c(\hat{t}))\right) G^{\prime}(\hat{t}) d \hat{t}
$$

where

$$
G^{\prime}(\hat{t})=\lambda\left[F(c(\hat{t}))+f(c(\hat{t})) c^{\prime}(\hat{t}) \hat{t}\right] e^{-\lambda F(c(\hat{t})) \hat{t}} \text { for each } \hat{t} \in[0, \infty)
$$

Then $\tilde{G}\left(t \mid t_{2}\right) \geq G(t)$ for all $t$, with strict inequality for $t>t_{2}$, that is, the distribution $\tilde{G}$ stochastically dominates the distribution $G$. Since $\left(\frac{v}{r}-E_{F}(c(\hat{t}))\right)$ is decreasing with $\hat{t}$, this implies that $B(0, c, \lambda)<B\left(t_{2}, \tilde{c}, \lambda\right)$. 
Claim 6 Let $c:\left(t_{1}, \infty\right) \rightarrow \mathbf{R}_{+}$be the function that maximizes $B(0, c, \lambda)$. Then $c$ is not decreasing on any interval.

Proof: Suppose to the contrary that $c$ is decreasing on a domain $\left[0, t_{2}\right]$. (The same argument works for any domain where $c$ is decreasing.) Define a threshold function $\tilde{c}$ : $(0, \infty) \rightarrow \mathbf{R}_{+}$by

$$
\tilde{c}(\hat{t})=c\left(\hat{t}+t_{2}\right) \text { for all } t \in[0, \infty)
$$

Thus, the function $\tilde{c}$ is identical to the optimizing function $c$ as $c$ is defined from $t_{2}$ forward, but it is shifted back to start at 0 instead of $t_{2}$. We will show that $B(0, c, \lambda)<B\left(t_{2}, c, \lambda\right)=$ $B(0, \tilde{c}, \lambda)$, which contradicts the fact that $c$ is optimal from time 0 . Using (13), the first inequality holds because $c$ is assumed optimal and decreasing, so $B$ is increasing. We show the equality.

$$
\begin{aligned}
B\left(t_{2}, c, \lambda\right) & =\int_{t_{2}}^{\infty} e^{-r\left(\hat{t}-t_{2}\right)}\left(\frac{v}{r}-E_{F}(c(\hat{t}))\right) \lambda F(c(\hat{t})) e^{-\int_{t_{2}}^{\hat{t}} \lambda F(c(t)) d t} d \hat{t} \\
& =\int_{t_{2}}^{\infty} e^{-r\left(\hat{t}-t_{2}\right)}\left(\frac{v}{r}-E_{F}\left(\tilde{c}\left(\hat{t}-t_{2}\right)\right)\right) \lambda F\left(\tilde{c}\left(\hat{t}-t_{2}\right)\right) e^{-\int_{0}^{\hat{t}-t_{2}} \lambda F(\tilde{c}(x)) d x} d \hat{t} \\
& =\int_{0}^{\infty} e^{-r t}\left(\frac{v}{r}-E_{F}(\tilde{c}(t))\right) \lambda F(\tilde{c}(t)) e^{-\int_{0}^{t} \lambda F(\tilde{c}(x)) d x} d t \\
& =B(0, \tilde{c}, \lambda)
\end{aligned}
$$

\subsection{Proof of Proposition 4}

We first show in Claim 7 that the optimal $c$ is either increasing everywhere, as we wish to show, or there exists $t_{1} \geq 0$ such that $c$ is increasing for $t<t_{1}$ and decreasing for $t>t_{1}$.

Claim 7 If $c$ maximizes $\tilde{B}(0, \cdot, \tilde{h})$, and if $c^{\prime}(t)=0$ at some $t$, then $c^{\prime \prime}(t)<0$. (The optimal $c$ can have at most one point where $c^{\prime}=0$, and at that point, $c$ is concave.)

Proof: We will use the derivative of $\tilde{B}$.

$$
\frac{d}{d t} \tilde{B}(t, c, \tilde{h})=\left[\frac{v}{r}-c(t)\right] r+\left[E_{F}(\tilde{c}(t))-c(t)\right] E(\lambda \mid t, \tilde{c}) \mathcal{F}(t \mid \tilde{c})
$$

If $c$ is optimal, it follows from (15) that $c^{\prime}(t)=0 \Longleftrightarrow \frac{d}{d t} \tilde{B}(t, c, \tilde{h})=0$ and $c^{\prime \prime}(t)<0 \Longleftrightarrow$ $\frac{d^{2}}{d t^{2}} \tilde{B}(t, c, \tilde{h})>0$. If $c^{\prime}(t)=0$ then $(d / d t) \mathcal{F}(t \mid \tilde{c})=0$ and $(d / d t)\left[E_{F}(\tilde{c}(t))-c(t)\right]=0$. 
But by Lemma 1, $(d / d t) E(\lambda \mid t, \tilde{c})<0$. Because $\left[E_{F}(\tilde{c}(t))-c(t)\right]<0$, this proves that $\frac{d^{2}}{d t^{2}} \tilde{B}(t, c, \tilde{h})>0$ and $c^{\prime \prime}(t)<0$.

Suppose, then, that there exists $t_{1}$ such that for $t>t_{1}, c$ is decreasing and $\tilde{B}$ is increasing. There are no relevant banked ideas at $t_{1}$. An idea with cost $c<c\left(t_{1}\right)$ would already have been used. An idea with cost $c>c\left(t_{1}\right)$ will never be used at any $t>t_{1}$ because $c>c\left(t_{1}\right)>c(t)$. Therefore, banking is irrelevant after $t_{1}$, and the stochastic process beginning at $t_{1}$ is exactly the same as when ideas are not banked. Let the "initial" beliefs at $t_{1}$ be $\tilde{h}_{1}$. Then, maintaining the hypothesis that $c$ is decreasing for $t>t_{1}$ (and $\tilde{B}$ is increasing), it holds that $\tilde{B}(t, c, \tilde{h})=\tilde{V}\left(t, c, \tilde{h}_{1}\right)$ for $t>t_{1}$. But we already showed in Proposition 2 that $\tilde{V}$ is decreasing, not increasing, which is a contradiction. This completes the proof.

\subsection{Proof of Proposition 5}

It is enough to show that $\tilde{B}(t, c, \tilde{h})>\tilde{V}(t, c, \tilde{h})$ for every increasing threshold function $c$. Define functions $g, \tilde{Q}_{V}$ and $\tilde{Q}_{B}$ by

$$
\begin{gathered}
g(\hat{t} \mid c)=e^{-r(\hat{t}-t)}\left(\frac{v}{r}-E_{F}(c(\hat{t}))\right) \\
\tilde{Q}_{V}(\hat{t} \mid c)=\int_{0}^{\infty} \tilde{h}(\lambda) e^{-\Lambda(\hat{t}, c)} d \lambda \\
\tilde{Q}_{B}(\hat{t} \mid c)=\int_{0}^{\infty} \tilde{h}(\lambda) e^{-\lambda F(c(\hat{t})) \hat{t}} d \lambda \\
\tilde{V}(t, c, \tilde{h})=\int_{t}^{\infty} \int_{0}^{\infty} g(\hat{t} \mid c) \lambda F(c(\hat{t})) e^{-[\Lambda(\hat{t}, c)-\Lambda(t, c)]} h(\lambda \mid t, c) d \lambda d \hat{t} \\
=\frac{1}{\tilde{Q}_{V}(t \mid c)} \int_{t}^{\infty} \int_{0}^{\infty} g(\hat{t} \mid c) \lambda F(c(\hat{t})) \tilde{h}(\lambda) e^{-\Lambda(\hat{t}, c)} d \lambda d \hat{t} \\
\tilde{Q}_{V}(t \mid c) \\
=\frac{1}{\tilde{Q}_{V}(t \mid c)} \int_{t}^{\infty} g(\hat{t} \mid c) F(c(\hat{t})) \int_{0}^{\infty} \lambda \tilde{h}(\lambda) e^{-\Lambda(\hat{t}, c)} d \lambda d \hat{t}
\end{gathered}
$$




$$
\begin{aligned}
\tilde{B}(t, c, \tilde{h}) & =\int_{t}^{\infty} \int_{0}^{\infty} g(\hat{t} \mid c) \lambda \mathcal{F}(\hat{t} \mid c) e^{-\lambda F(c(\hat{t})) \hat{t}+\lambda F(c(t)) t} h(\lambda \mid t, c) d \lambda d \hat{t} \\
& =\frac{1}{\tilde{Q}_{B}(t \mid c)} \int_{t}^{\infty} \int_{0}^{\infty} g(\hat{t} \mid c) \lambda \mathcal{F}(\hat{t} \mid c) \tilde{h}(\lambda) e^{-\lambda F(c(\hat{t})) \hat{t}} d \lambda d \hat{t} \\
& =\frac{1}{\tilde{Q}_{B}(t \mid c)} \int_{t}^{\infty} g(\hat{t} \mid c) \mathcal{F}(\hat{t} \mid c) \int_{0}^{\infty} \lambda \tilde{h}(\lambda) e^{-\lambda F(c(\hat{t})) \hat{t}} d \lambda d \hat{t} \\
& =\frac{1}{\tilde{Q}_{B}(t \mid c)} \int_{t}^{\infty} g(\hat{t} \mid c) \frac{d}{d \hat{t}}\left[1-\tilde{Q}_{B}(\hat{t} \mid c)\right] d \hat{t}
\end{aligned}
$$

At each $\hat{t}>0, e^{-\Lambda(\hat{t}, c)}>e^{-\lambda F(c(\hat{t})) \hat{t}}$. Therefore,

$$
\tilde{Q}_{V}(\hat{t} \mid c)=\int_{0}^{\infty} \lambda \tilde{h}(\lambda) e^{-\Lambda(\hat{t}, c)} d \lambda>\int_{0}^{\infty} \lambda \tilde{h}(\lambda) e^{-\lambda F(c(\hat{t})) \hat{t}} d \lambda=\tilde{Q}_{B}(\hat{t} \mid c)
$$

and $1-\tilde{Q}_{V}(\cdot \mid c)$ stochastically dominates $1-\tilde{Q}_{B}(\cdot \mid c)$. Since $g(\cdot)$ is decreasing when $c$ is increasing, it follows that $\tilde{Q}_{B}(t \mid c) \tilde{B}(t, c, \tilde{h})>\tilde{Q}_{V}(t \mid c) \tilde{V}(t, c, \tilde{h})$.

But since $\tilde{Q}_{B}(t \mid c) / \tilde{Q}_{V}(t \mid c)<1$, it follows that $\tilde{B}(t, c, \tilde{h})>\tilde{V}(t, c, \tilde{h})$.

\subsection{Proof of Remark 1}

(a) Let $n$ be the number of potential recipients. Suppose that each recipient believes that each other recipient follows an investment strategy described by a cost threshold function $c$. At date $t$, some recipients have received ideas. If a recipient received a single idea at, say $\tilde{t} \leq t$, the recipient takes this into account in forming his belief on $\lambda$. In a population of size $n$, with individual arrival rate $\lambda / n$, the agent's belief on $\lambda$ is given by the following posterior density:

$$
g_{n}(\hat{\lambda} \mid \tilde{t}, t, c)=\frac{\frac{\hat{\lambda}}{n} e^{-\frac{\hat{\lambda}}{n} \tilde{t}} e^{-\left(\frac{n-1}{n}\right) \hat{\lambda} \int F(c(t)) d t} \tilde{h}(\hat{\lambda})}{\int \frac{\lambda}{n} e^{-\frac{\lambda}{n} \tilde{t}} e^{-\left(\frac{n-1}{n}\right) \lambda \int F(c(t)) d t} \tilde{h}(\lambda) d \lambda}=\frac{\hat{\lambda} e^{-\left(\frac{n-1}{n}\right) \hat{\lambda} \int F(c(t)) d t} \tilde{h}(\hat{\lambda})}{\int \lambda e^{-\frac{(\lambda-\hat{\lambda})}{n} \tilde{t}} e^{-\left(\frac{n-1}{n}\right) \lambda \int F(c(t)) d t} \tilde{h}(\lambda) d \lambda}
$$

Let $r$ be the limit density function as $n \rightarrow \infty$ :

$$
r(\hat{\lambda} \mid t, c)=\frac{\hat{\lambda} e^{-\hat{\lambda} \int F(c(t)) d t} \tilde{h}(\hat{\lambda})}{\int \lambda e^{-\lambda \int F(c(t)) d t} \tilde{h}(\lambda) d \lambda}
$$

The limit distribution does not depend on $\tilde{t}$, as asserted in part (a).

Further, the probability of receiving more than one idea has a second-order effect, and we therefore ignore it. The numerator in the following expression is the probability of 
receiving two or more ideas by time $t$, and the denominator is the probability of receiving one or more ideas by time $t$. Using L'Hopital's rule, the ratio converges to zero.

$$
\lim _{n \rightarrow \infty} \frac{\left[1-e^{-\frac{\lambda}{n} t}-\left(\frac{\lambda}{n} t\right) e^{-\frac{\lambda}{n} t}\right]}{\left[1-e^{-\frac{\lambda}{n} t}\right]} \rightarrow 0
$$

This concludes part (a).

(b) Nevertheless, the recipients are more optimistic about $\lambda$ than the planner. When the planner believes that $c$ describes the recipients' investment behavior, the density of the planner's posterior is

$$
h(\hat{\lambda} \mid t, c)=\frac{e^{-\hat{\lambda} \int F(c(t)) d t} \tilde{h}(\hat{\lambda})}{\int e^{-\lambda \int F(c(t)) d t} \tilde{h}(\lambda) d \lambda}
$$

The ratio of the densities, $r(\hat{\lambda} \mid t, c) / h(\hat{\lambda} \mid t, c)$, is proportional to $\lambda$. This implies that the recipients, as opposed to the planner, place higher weight on higher $\lambda$, that $r$ stochastically dominates $h$, and that $r$ has a higher expected value. It is instructive to show the latter directly.

Divide both numerator and denominator of $r$ by $\int e^{-\lambda \int F(c(t)) d t} \tilde{h}(\lambda) d \lambda$ :

$$
r(\hat{\lambda} \mid t, c)=\frac{\frac{\hat{\lambda} e^{-\hat{\lambda} \int F(c(t)) d t} \tilde{h}(\hat{\lambda})}{\int e^{-\lambda \int F(c(t)) d t} \tilde{h}(\lambda) d \lambda}}{\frac{\int \lambda e^{-\lambda \int F(c(t)) d t} \tilde{h}(\lambda) d \lambda}{\int e^{-\lambda \int F(c(t)) d t} \tilde{h}(\lambda) d \lambda}}=\frac{\hat{\lambda} e^{-\hat{\lambda} \int F(c(t)) d t} \tilde{h}(\hat{\lambda})}{E_{h}(\lambda \mid t, c) \int e^{-\lambda \int F(c(t)) d t} \tilde{h}(\lambda) d \lambda}
$$

Let $E_{h}(\lambda \mid t, c)$ and $E_{h}\left(\lambda^{2} \mid t, c\right)$ be the expected values with respect to the planner's posterior belief, and let $\operatorname{Var}_{h}(\lambda)$ be the variance of the planner's belief. Now consider the expected value of $\lambda$ with respect to the recipients' belief instead of the planner's belief:

$$
\begin{aligned}
E_{r}(\lambda \mid t, c) & =\int \hat{\lambda} r(\hat{\lambda} \mid t, c) d \hat{\lambda}=\frac{\int \hat{\lambda}^{2} \frac{e^{-\hat{\lambda} \int F(c(t)) d t} \tilde{h}(\hat{\lambda})}{\int e^{-\lambda \int F(c(t)) d t} \hat{h}(\lambda) d \lambda} d \hat{\lambda}}{E_{h}(\lambda \mid t, c)}=\frac{E_{h}\left(\lambda^{2} \mid t, c\right)}{E_{h}(\lambda \mid t, c)} \\
& =\left(\frac{1}{E_{h}(\lambda \mid t, c)}\right)\left(\operatorname{Var}_{h}(\lambda)+E_{h}(\lambda \mid t, c)^{2}\right)=\frac{\operatorname{Var}_{h}(\lambda)}{E_{h}(\lambda \mid t, c)}+E_{h}(\lambda \mid t, c)
\end{aligned}
$$

Thus, $E_{r}(\lambda \mid t, c)>E_{h}(\lambda \mid t, c)$.

Part (c) follows from (17). 


\section{References}

[1] Choi, J. P. 1991. "Dynamic R\&D Competition under 'Hazard Rate' Uncertainty," RAND Journal of Economics, 22, 596-610

[2] Denicolo, V. and L. Franzoni. 2004. "Patents, Secrets, and the First Inventor Defense," Journal of Economics and Management Strategy, 13, 517-538.

[3] Dixit, A. and R. Pindyck. 1994. Investment under Uncertainty. Princeton, NJ: Princeton University Press.

[4] Erkal, N. 2005. "The Decision to Patent, Cumulative Innovation, and Optimal Policy," International Journal of Industrial Organization, 23(7-8), 535-562.

[5] Erkal, N. and S. Scotchmer. 2007. "Scarcity of Ideas and Options to Invest in R\&D," University of California, Berkeley, Department of Economics, Working Paper 07-348.

[6] Hopenhayn, H., G. Llobet, and M. Mitchell. 2006. "Rewarding Sequential Innovators: Patents, Prizes, and Buyouts." Journal of Political Economy 114:1041-1068.

[7] McCall, B. P. and J. J. McCall. 2008 Economics of Search. New York: Routledge Press.

[8] MacDonald, R. and D. Siegel. 1986. "The Value of Waiting to Invest," Quarterly Journal of Economics, 101, 707-728.

[9] Malueg, D. A. and S. O. Tsutsui. 1997. "Dynamic R\&D Competition with Learning," RAND Journal of Economics 28, 751-772.

[10] O’Donoghue, T., Scotchmer, S. and Thisse, J.-F. 1998. "Patent Breadth, Patent Life and the Pace of Technological Progress," Journal of Economics and Management Strategy, 7, 1-32.

[11] Reinganum, J. 1989. "The Timing of Innovation: Research, Development and Diffusion," in R. Schmalensee and R. D. Willig, eds., Handbook of Industrial Organization, Amsterdam: Elsevier. 
[12] Scotchmer, S. 1999. "On the Optimality of the Patent Renewal System," RAND Journal of Economics, 30, 131-196.

[13] Scotchmer, S. 2004. Innovation and Incentives. Cambridge, MA: MIT Press.

[14] Snyder, D. L. and M. I. Miller. 1991. Random Point Processes in Time and Space. New York: Springer-Verlag.

[15] Weeds, H. 2002. "Strategic Delay in a Real Options Model of R\&D Competition," Review of Economic Studies, 69, 729-747.

[16] Wright, B. D. 1983. "The Economics of Invention Incentives: Patents, Prizes and Research Contracts." American Economic Review 73:691-707. 